\title{
Wavelength dependence of polarimetric and phase-shift characterization of a liquid crystal on silicon display
}

\author{
A. Lizana \\ angel.lizana@uab.cat \\ A. Márquez \\ I. Moreno \\ C. Iemmi \\ J. Campos
}

M. J. Yzuel
Dept. Física, Univ. Autónoma de Barcelona, 08193 Bellaterra, Spain

Dept. Física, Ing. Sistemas y T. Señal, Univ. Alicante, 03080 Alicante, Spain

Dept. C. Materiales, Óptica y T. Electrónica, Univ. Miguel Hernández, Elche, Spain

Dept. Física, Univ. Buenos Aires, Argentina

Dept. Física, Univ. Autónoma de Barcelona, 08193 Bellaterra, Spain

Dept. Física, Univ. Autónoma de Barcelona, 08193 Bellaterra, Spain

We present a full characterization of a liquid-crystal-on-silicon (LCOS) display, including a polarimetric analysis based on the Mueller-Stokes formalism, and a phase shift modulation calibration. Results for different wavelengths are compared. The goal of this work is two fold. On one side, previous papers dealing with the illumination wavelength $633 \mathrm{~nm}$ have shown that LCoS produce a non negligible amount of depolarized light. This may have a negative impact in certain applications. Here we want to establish how this depolarization varies with the wavelength. Diattenuation is also evaluated. On the other side, to use the LCoS as a spatial light modulator (SLM) we need to obtain optimal configurations enabling for phase-only or for amplitude-only modulation. Here we show how phase-only modulation is obtained, and how it evolves with the wavelength. In principle, the phase modulation depth increases and the energy throughput may also be increased for shorter wavelengths. However, these phase-only configurations may be partially degraded by the presence of depolarization at certain gray levels. Thus, the Mueller-Stokes formalism is necessary to get a full picture of the performance exhibited by the LCoS at each wavelength. [DOI: 10.2971/jeos.2008.08012]

Keywords: Mueller matrix, liquid crystal display, spatial light modulator, phase-only modulation, polarization

\section{INTRODUCTION}

Liquid crystal displays (LCD) have found widespread use in applications requiring spatial light modulation, such as holographic data storage [1], diffractive optics [2] and optical metrology [3]. They can be used as amplitude-only or as phase-only spatial light modulators (SLM) when properly selecting the input and output polarization configurations [4, 5]. In recent years new liquid crystal on silicon (LCoS) based displays have attracted considerable interest due to their high resolution and excellent fill factor [6]-[9]. They are reflective displays which enable for an increase in the dynamic modulation range compared to transmissive displays, for a given width of the liquid crystal layer. This property is especially important in applications requiring phase-shift modulation of the incident wavefront.

Recent publications dealing with LCoS displays [10, 11] have shown the existence of certain effective depolarization in the light reflected by LCoS. In particular, in reference [11] measurements show up to a $10 \%$ of depolarized light for some gray levels and for certain incident states of polarization. We demonstrated that the origin of this depolarization effect is mainly the temporal fluctuation of the state of polarization
(SOP) reflected by the LCoS during the frame period. This fluctuation is due to instabilities in the electrical signal addressed. Specific details of the electrical signal addressing can be found in [12], where it is shown that present LCOS are digitally addressed, which causes subframe flickering if the time length of the pulses is not properly optimized by the driver electronics. When performing time-resolved polarimetric measurements the reflected SOP in every instant of time is totally polarized but the output SOP changes with time. This implies that the averaged SOP shows certain degree of depolarization.

The presence of depolarized light suggests the use of the Mueller-Stokes formalism instead of the Jones or the Berreman formalisms to describe the performance of the device. Due to the reflected SOP temporal fluctuations, developing a microscopic model to describe the LCoS display becomes complicated, and we have chosen the Mueller matrix as a tool to describe the LCoS display in a more phenomenological way. In [11] the LCoS was characterized using the MuellerStokes formalism for the wavelength $\lambda=633 \mathrm{~nm}$. Once the Mueller matrix is obtained, SOP and the degree of polariza- 
tion (DoP) for the light reflected by the LCoS can be obtained. The knowledge of the Mueller matrix also allowed us to obtain optimal configurations of the external polarization elements in terms of the intensity transmission for the whole range of applied gray levels. Under certain conditions these configurations also provide a large phase dynamic range, thus leading to phase-only modulation. In this work we extend this previous study [11] for other two shorter wavelengths, such as 532 and $458 \mathrm{~nm}$.

The interest of the wavelength extension of this polarimetric study is two fold. First, the modulation dynamic range increases as the wavelength is reduced. Thus, the phase modulation depth available increases too. As a consequence light throughput may be increased while maintaining a $360^{\circ}$ phase dynamic range in phase-only configurations, interesting for example in diffractive optics or in optical image processing applications. Furthermore, this study is useful for applications where the SLM is illuminated simultaneously with a set of discrete wavelengths covering the visible spectrum [13] or with a continuum broadband spectrum [14]. Last but not least, the analysis of the wavelength dependence of polarimetric parameters is especially interesting in the present case to learn how they are affected by the temporal fluctuations of the electrical signal.

In this paper we extend the results presented in [11] by using different wavelengths and comparing their effect on the depolarization and phase modulation. Certain working regimes are especially interesting for applications. In this paper, we focus on phase-only modulation, where it is interesting to obtain constant amplitude, with the maximum intensity throughput, a $360^{\circ}$ phase modulation depth, and with no coupled depolarized light. In Section 2 we show results for the Stokes-Mueller characterization, with an emphasis on depolarization. In Section 3, the evolution with wavelength of phase-only modulation configurations is analyzed. Eventually, the main conclusions are given in Section 4.

\section{STOKES-MUELLER CHARACTERIZATION AS A FUNCTION OF WAVELENGTH}

In this section we extend to the wavelengths $532 \mathrm{~nm}$ (diode laser) and $458 \mathrm{~nm}$ (Ar air-cooled laser) the measurements done for $633 \mathrm{~nm}$ (He-Ne laser) in [11]. The LCoS under analysis is a Philips model X97c3A0, sold as the kit LC-R2500 by Holoeye. The LC-R2500 is a $2.46 \mathrm{~cm}$ diagonal monochrome reflective LCoS of the $45^{\circ}$ twisted nematic type, with XGA resolution (1024 x 768 pixels), with digital data input and digitally controlled gray scales with 256 gray levels. The pixels are square with a pixel center to center separation of $19 \mu \mathrm{m}$ and a fill factor of $93 \%$.

In [11] we showed that the origin for the depolarized light is the temporal fluctuation of the SOP reflected by the LCoS. These fluctuations depend on the gray level addressed and on the configuration for the external polarization devices in front and behind the LCoS. To this goal we measured the instantaneous optical intensity transmitted by the LCoS. In Figure1 we plot the optical intensity registered by a digital oscilloscope
(Tektronix TDS3012B), as a function of time and for different gray levels addressed to the LCoS display. These measurements have been performed using the LCoS sandwiched between two polarizers at $0^{\circ}$ of the laboratory vertical and with the $633 \mathrm{~nm}$ wavelength. We see that at the low and high gray level ranges there are small fluctuations as a function of time (or almost null fluctuations, for instance at the 0 gray level). However, at the gray level range around 200 there are strong fluctuations. Therefore, we see the strong dependence of the fluctuation phenomena with the gray level addressed to the LCoS display.

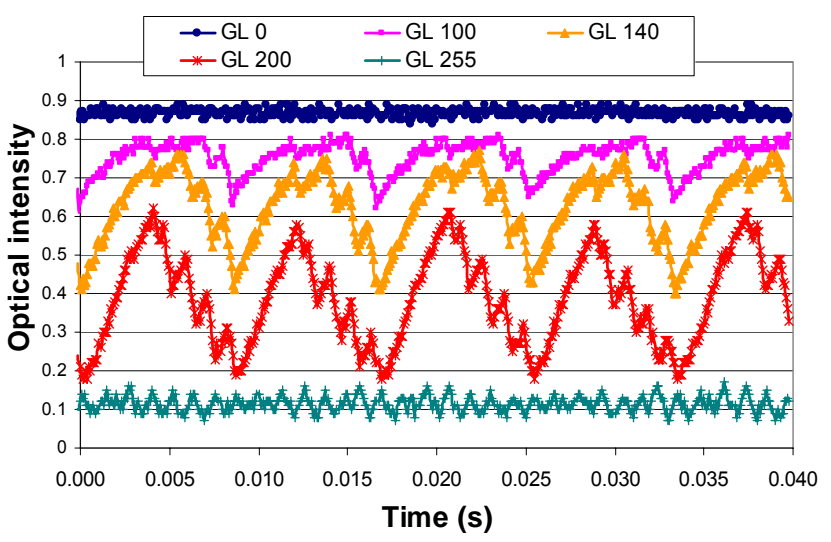

FIC. 1 Optical intensity registered with the digital oscilloscope for the $633 \mathrm{~nm}$ wave length as a function of time and for several gray levels addressed to the LCoS display.

In order to show the existence of these fluctuations at different wavelengths, we have repeated these measurements at 532 $\mathrm{nm}$ and $458 \mathrm{~nm}$. In Figure 2 we plot the signal registered by the oscilloscope, normalized to its maximum value for each of the three wavelengths and at gray level 200, where the fluctuations are larger. In order to emphasize that these fluctuations are caused by the display we want to state that during the measurements the intensity of the light source remained constant. The measurements have been done using only polarizers (as we show next, optimized modulation responses are obtained by using elliptically polarized light configurations). We have oriented the polarizers in such a way that the fluctuations of the signal registered by the oscilloscope had a large amplitude (angles for the polarizers are given in the caption). We see that the amplitude of the oscillations of the signals practically equals the lengths for the vertical axis. The fluctuations present a periodicity, with a frequency of about $60 \mathrm{~Hz}$ (period $\approx 17 \mathrm{~ms}$ ) and a subfrequency of about $120 \mathrm{~Hz}$ (period $\approx 8 \mathrm{~ms}$ ). These periodicities are probably related with the frame rate and the field rate of the incoming video signal to the LCoS panel (technical specifications do not offer these details).

The Mueller matrix formalism for polarization is well adapted to describe the performance of polarization devices exhibiting depolarization. To obtain a full polarimetric characterization of the LCoS we proceed with the measurement of the Stokes parameters for the SOP of the light reflected by the LCoS for a series of input SOPs. The set-up used for the measurements is shown in Figure 3. A polarization state generator (PSG) and a polarization state detector (PSD) are located in 


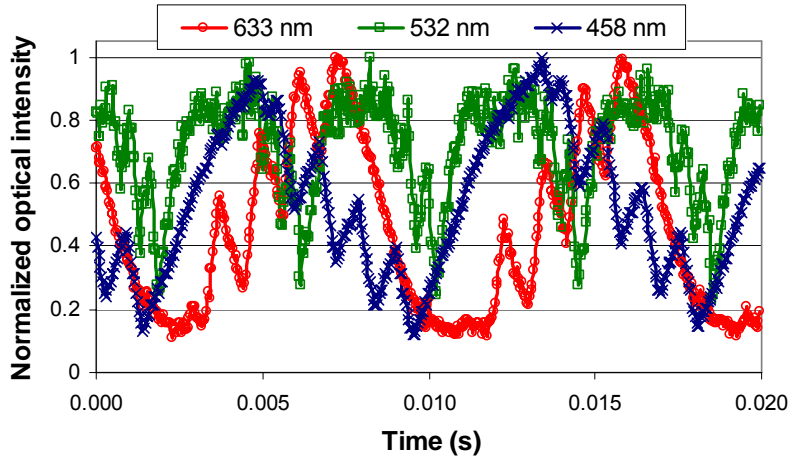

FIG. 2 Optical signal registered with the digital oscilloscope for the three wavelengths at gray level 200 and with only polarizers. The configurations for the input and output polarizers are $\left(45^{\circ}, 60^{\circ}\right)$ for $633 \mathrm{~nm},\left(45^{\circ}, 45^{\circ}\right)$ for $532 \mathrm{~nm}$, and $\left(45^{\circ}, 70^{\circ}\right)$ for 458 $\mathrm{nm}$.

front and behind the LCOS respectively. They are composed of a linear polarizer and a quarter waveplate, and they are mounted on rotating holders to allow the polarimetric characterization [15]. Specifically, we generate and detect 6 different SOPs: linearly polarized along the vertical, horizontal, at $45^{\circ}$ and at $-45^{\circ}$, and right-handed and left-handed circularly polarized light (labelled in the following as X, Y, 45, -45 , R and L, respectively). In these experiments we measure the intensity for the light after the PSD. The laser beam impinges at nonperpendicular incidence onto the LCoS, in order to separate the incident and the reflected beams of light. The angle of incidence with respect to the normal of the LCoS is $2^{\circ}$, i.e. quasiperpendicular incidence. Additional details for the setup and applied methodology can be found in [11]. We note that the accuracy of the measured Mueller matrix for the LCoS has been verified, as in [11]. We check its validity to predict the output polarization state for an arbitrary incident SOP by comparing the experimental measurements and the predicted output SOP for a given input SOP. In particular, as the incident SOP we consider linearly polarized light at $30^{\circ}$ from the vertical of the lab. Experimental and calculated values for the Stokes parameters and DoP of the light reflected by the LCoS show a very good agreement as in [11]. In Section 3 we will show explicitly the good agreement between experimental data and values calculated using the measured Mueller matrix.

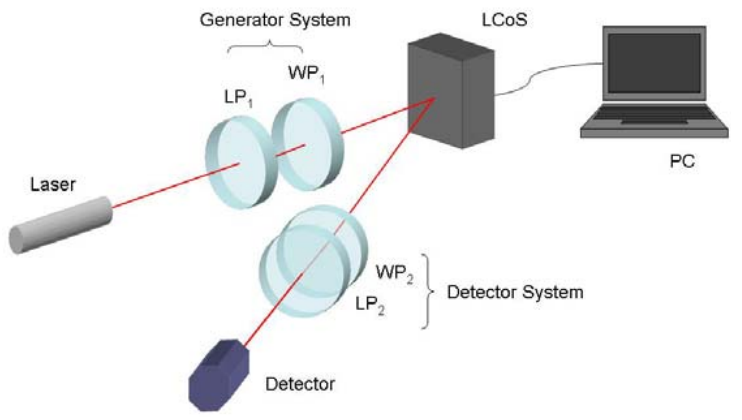

FIG. 3 Experimental set-up.

From the Stokes measurements we can obtain both the degree of polarization (DoP) of the reflected light, and other polarimetric data as the diattenuation coefficient as given in [11, 15]. In Figure 4 we show the DoP as a function of the gray level, for the six different input SOPs (X, Y, 45, -45 , R and L), respectively for the wavelengths 633, 532 and $458 \mathrm{~nm}$ in Figures 4a, $\mathrm{b}$ and $\mathrm{c}$. Note that the range for the vertical scale is different for the three wavelengths. For all three wavelengths the DoP varies both with the gray level and with the incident SOP. If we compare the three wavelengths we see that the shorter the wavelength the smaller the DoP. The depolarization effect origin is the temporal variation of the molecules orientation originated from the electrical signal fluctuation. The changes in the orientation cause a variation of the output polarization state. These polarization changes become stronger as the wavelength is reduced. In particular, for $458 \mathrm{~nm}$ the DoP is lower than 0.6 in the range around gray level 200. Something interesting to note at this wavelength is that, opposed to the other incident SOPs, the DoP stays close to one along most of the gray level range for input SOPs linear X and Y. To understand this result we should take into account that the matrix describing a reflective LCD is equivalent to a rotated linear retarder $[16,17]$. In particular, for the LCoS in this work and for $458 \mathrm{~nm}$, we have calculated that the eigenpolarizations for the equivalent retarder are mainly along the vertical and horizontal of the lab, thus they coincide with the input SOPs X and Y. It can be expected that the temporal fluctuations in the LCoS do not affect the SOP for these two input states.

In Figure $4 \mathrm{~d}$ we represent the averaged DoP as a function of the gray level and for each of the three wavelengths. This averaged DoP is defined as the arithmetic average calculated from the 6 values measured for the 6 incident SOPs shown in Figures $4 \mathrm{a}, \mathrm{b}$ and c. Since these 6 SOPs are equally distributed along the Poincaré sphere, this average shows the tendency of the temporal fluctuations in the LCoS to depolarize light. It can be clearly seen that the DoP decreases for shorter wavelengths, especially for gray levels larger than 100. If we divide the minimum DoP in the graph by the corresponding wavelength we obtain approximately equal rations (about 1.4). Thus, as a rough estimation we can say that the DoP is linearly related with the wavelength. We note that the uncertainty in the calculated DoP, due to the \pm 0.02 uncertainty in the Stokes parameters, can be estimated as \pm 0.04 in the various DoP results presented along this work. This uncertainty may lead in some configurations and certain gray levels to values of DoP slightly over 1, situations where we can consider that the light is fully polarized.

The measurements for the Stokes parameters for the 6 input SOPs also allow calculating the diattenuation coefficients for the LCoS [11]. We do not show here these results but we note that the magnitude of the diattenuation is in all the cases nearly equal or lower than 0.1 , thus we can say that it is not important and the LCoS for the three wavelengths can be basically considered as the combination of a retarder and a depolarizer as it resulted in [18].

\section{OPTIMAL CONFIGURATIONS}

In this section we have analyzed the evolution with wavelength of phase-only modulation configurations. Since the birefringence of the liquid crystal is wavelength sensitive, changing the wavelength of the incoming light should result 

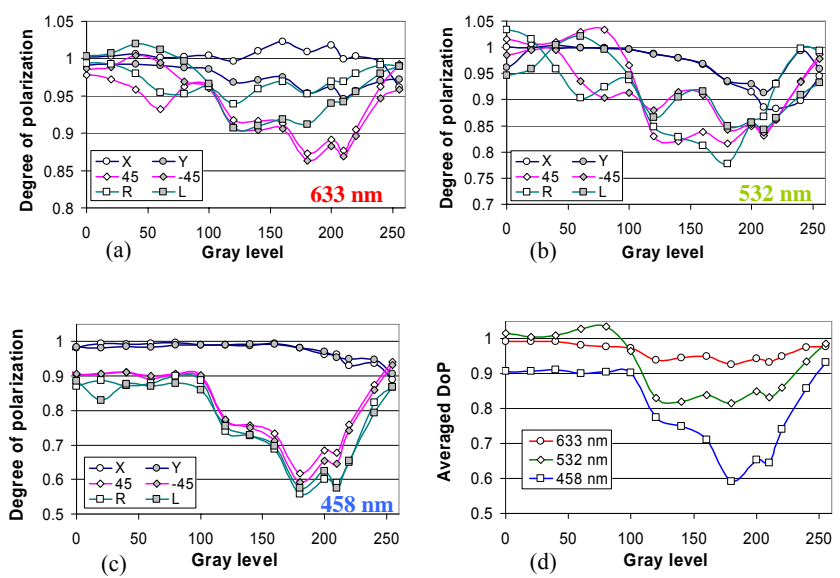

FIG. 4 Degree of polarization (DoP) for the 6 inputs SOPs. (a) $\lambda=633 \mathrm{~nm}$, (b) $\lambda=532$ $\mathrm{nm}$, (c) $\lambda=458 \mathrm{~nm}$, and (d) averaged DoP.

in a different response. The use of shorter wavelengths leads to larger phase modulations depths. This may be a crucial issue in applications requiring a phase-only modulation where the diffraction efficiency is directly related to the phase depth.

By means of the measured Mueller matrix for the LCoS as a function of the gray level we are able to predict the intensity, the state and the degree of polarization of the light reflected for an arbitrary polarization configuration. However, the Mueller matrix of the LCoS display depends on the gray level and on the wavelength. For this reason the measurement of the experimental matrix for every gray level and every wavelength has been required.

We have used the prediction capability of the obtained matrices to find configurations giving constant intensity response. The experience tells us that if a configuration gives almost constant intensity response, this configuration or its biorthogonal [11] will give a large phase shift. According to this strategy we have found configurations giving excellent results in terms of constant intensity and with a large phase shift modulation depth for the three wavelengths. The obtained configurations have been found by means of an iterative optimization procedure based on a computational search of the ellipticity and orientation, of the generator and detector systems, leading to a minimum value of an appropriate figure of merit. In general, the result of this computational search may correspond to a local minimum. For that reason we performed several computational searches, for every wavelength used, starting from different input and output ellipticity and orientation configurations. We have found that several configurations of the generated and detected SOP give similar results (i.e. there are several local minimums with similar value of the quality criteria). Therefore, we can not relate the "optimum" SOPs for different wavelengths. The optimization procedure is explained in more detail in [11].

First, we have optimized the system response when using $633 \mathrm{~nm}$ wavelength and elliptically polarized light, obtaining some configurations giving large phase shift and constant intensity response. As we want to study thoroughly the evolution of the phase shift and the constancy for the intensity modulation with the wavelength, we have set the configuration giving the best results for $633 \mathrm{~nm}$ and we have tested it when using $532 \mathrm{~nm}$ and $458 \mathrm{~nm}$. In the experiments we generate the elliptical polarization by means of two achromatic quarter waveplates. Thus the change in the wavelength does not modify the polarization configuration.

Figure 5a corresponds to $633 \mathrm{~nm}$, when an elliptical polarization configuration is selected to produce a phase-only response. A constant intensity response between the values 0.6 and 0.7 is obtained. We see an excellent agreement between theoretical (brown line) and experimental intensity values (brown spots). We have checked other configurations of polarizers and waveplates and the agreement between theoretical predictions and experimental results also was excellent. Moreover, the almost constant intensity response is accompanied with a phase modulation almost reaching $2 \pi$ (green squares). This result improves our previous results presented in [11], where the polarization configuration was selected to a phase-only modulation with maximum intensity transmission. The reduction in the mean value of the intensity modulation allows increasing the phase dynamic range up to $2 \pi$ radians. This situation improves the usual case in transmissive displays, for which it is necessary to use shorter wavelengths to obtain a similar response. In addition, depolarization is negligible, since the degree of polarization (DoP) (blue line) is maintained equal to one in the whole gray level range.

Figure $5 \mathrm{~b}$ and $\mathrm{c}$ show the results using $532 \mathrm{~nm}$ and $458 \mathrm{~nm}$ respectively when setting the same configuration which gave the best results for $633 \mathrm{~nm}$ (this is, Figure 5a). For these wavelengths, the configuration is not optimal, and the phase-only response is degraded by a coupled intensity modulation and by an increase of the depolarization for high gray levels. Simultaneously, the phase modulation depth increases, reaching almost $3 \pi$ for $458 \mathrm{~nm}$. However, for this wavelength the intensity transmission is far from being constant across the gray level, with a variation between the minimum and the maximum values of about 0.6 . We note that there are applications where it is important to have modulation regimes which show a similar modulation profile across a certain range of wavelengths, as given in $[13,14]$.
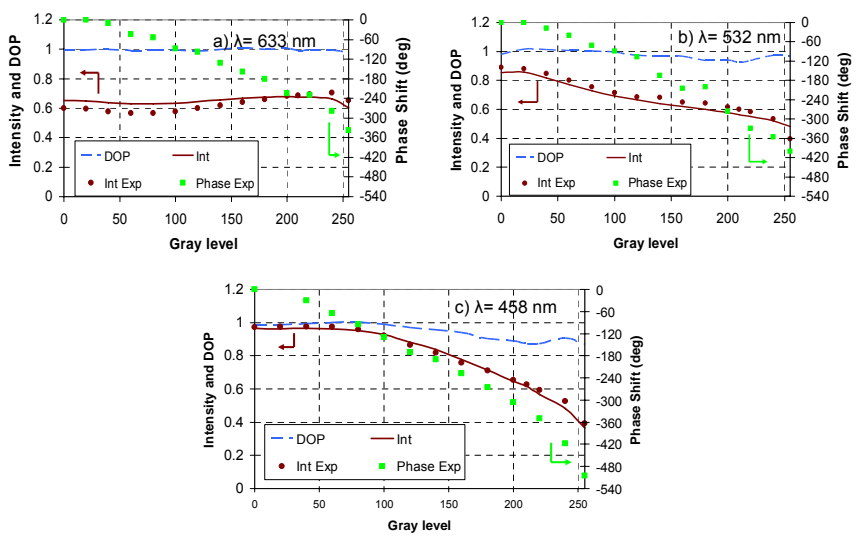

FIC. 5 Results for the same polarization configuration using different wavelengths. Brown line: theoretical intensity; brown spots: experimental intensity; green squares: experimental phase; discontinuous blue line: DOP. (a) $\lambda=633 \mathrm{~nm}$; (b) $\lambda=532 \mathrm{~nm}$; (c) $\lambda=458 \mathrm{~nm}$. 
As we want to obtain configurations giving pure phase modulation we have tried to solve the coupling between the intensity and phase modulations by optimizing the system response in each wavelength. In Figure 6 we see some obtained modulation results as a function of the gray level when optimizing the system response for $633 \mathrm{~nm}$ (Figure 6a), $532 \mathrm{~nm}$ (Figure $6 \mathrm{~b}$ ) and $458 \mathrm{~nm}$ (Figure $6 \mathrm{c}$ and $\mathrm{d}$ ).

Figure $6 \mathrm{~b}$ corresponds to a configuration optimized for 532 $\mathrm{nm}$, using an elliptical polarization light. We see an almost constant intensity response accompanied with a phase shift of $2 \pi$. Furthermore, the DoP is almost equal to one as a function of the gray level and we can consider that there is no unpolarized light. The profiles for the intensity and the phase shift modulations obtained in Figure $6 \mathrm{~b}$ are similar to the ones obtained in Figure 6a, when optimizing for 633 nm, but for 532 $\mathrm{nm}$ we obtain a higher average intensity value. It means that optimizing the response for $532 \mathrm{~nm}$ we can obtain similar values for phase shift that the ones obtained with $633 \mathrm{~nm}$ but also increase the values of intensity throughout the gray level range. Next, Figure $6 \mathrm{c}$ shows the system response corresponding to a configuration optimized for $458 \mathrm{~nm}$ and using elliptically polarized light. From Figure $6 \mathrm{c}$ we see an almost constant intensity response in the gray level range $0-240$ with intensity values up to 0.7 in all the range. Moreover, the constant intensity is accompanied in this range with a phase shift higher than $2 \pi$. This result improves the one obtained with the $633 \mathrm{~nm}$ because this configuration shows higher averaged intensity and phase shift. However, the degree of polarization reaches values about 0.9 at some gray levels and then there is approximately a $10 \%$ of unpolarized light. Finally, in order to obtain more phase shift, we have allowed in our optimization procedure to decrease the minimum intensity value when optimizing for $458 \mathrm{~nm}$ (seeFigure 6d). From Figure 6d we see an almost constant intensity response as a function of the gray level accompanied with a phase shift higher than $3 \pi$. However, there is again some unpolarized light in some gray level. Nevertheless, in terms of phase shift response, we see that using $458 \mathrm{~nm}$ we obtain more phase shift modulation than when using the previous two wavelengths.
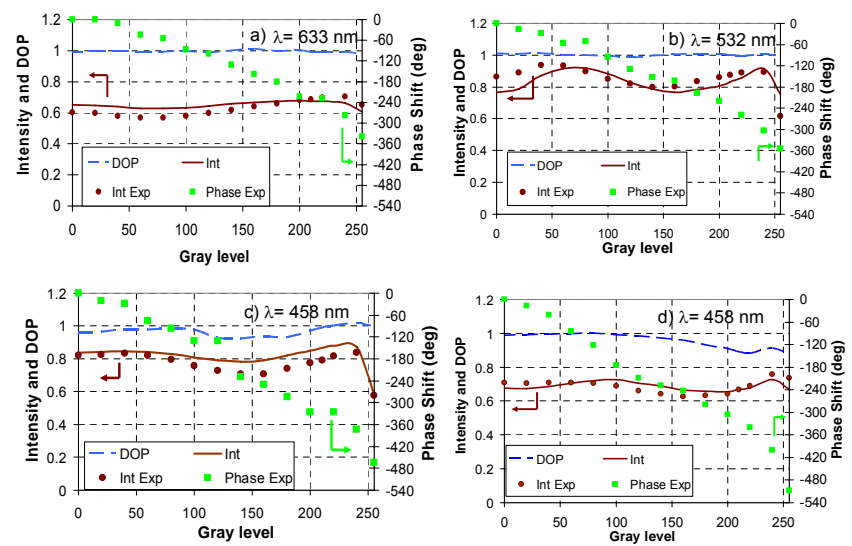

FIC. 6 Optimized intensity (brown line), theoretical DoP (blue line), experimental intensity (brown spots) and experimental phase-shift (green squares). (a) $\lambda=633 \mathrm{~nm}$, (b) $\lambda=532 \mathrm{~nm}$, (c) and (d) $\lambda=458 \mathrm{~nm}$.

The analysis of these results tells us that when using shorter wavelengths a higher phase shift is obtained, however the profile for the intensity modulation changes. Optimizing the system response in each case for the specific wavelength solves this problem and enables obtaining configurations giving almost a constant intensity response and a large phase shift modulation depth exceeding from $2 \pi$. Nevertheless, the appearance of non negligible values of unpolarized light must also be taken in account as we have shown in Section 2.

\section{CONCLUSIONS}

We have presented the results for the full characterization of an LCoS display for different wavelengths. The use of the Mueller-Stokes formalism enables to obtain polarimetric data that in the case of the present LCoS is very significant. In particular, the LCoS exhibits depolarized light, whose amount increases inversely proportional with the wavelength. At 458 $\mathrm{nm}$ the amount of depolarized light may be larger than a $40 \%$ at certain gray levels and for certain input states of polarization. In principle, shorter wavelengths may be interesting to obtain phase-only modulation with a high value for the intensity throughput. We see that at shorter wavelengths is specially required to use the Mueller-Stokes formalism since it allows evaluating the amount of depolarized light which may have a degradation impact in certain applications.

\section{ACKNOWLEDGMENTS}

We acknowledge financial support from the Spanish Ministerio de Educación y Ciencia (grants FIS2006-13037-C02-01 and FIS2006-13037-C02-02) and from Generalitat de Catalunya (grant ACI2003-42). C. Iemmi gratefully acknowledges the support of the Universidad de Buenos Aires and CONICET (Argentina).

\section{References}

[1] A. Márquez, S. Gallego, D. Méndez, M. L. Álvarez, E. Fernández, M. Ortuño, C. Neipp, A. Beléndez and I. Pascual, "Accurate control of a liquid crystal display to produce a homogenized Fourier transform for holographic memories" Opt. Lett. 32, 2511-2513 (2007).

[2] R. Tudela, E. Martín-Badosa, I. Labastida, S. Vallmitjana, I. Juvells and A. Carnicer, "Full complex Fresnel holograms displayed on liquid crystal devices" J. Opt. A: Pure Appl. Opt. 5, S189-S194 (2003).

[3] W. Osten, C. Kohler and J. Liesener, "Evaluation and application of spatial light modulators for optical metrology" Opt. Pura Apl. 38, 71-81 (2005).

[4] A. Márquez, C. Iemmi, I. Moreno, J. A. Davis, J. Campos and M. J. Yzuel, "Quantitative prediction of the modulation behavior of twisted nematic liquid crystal displays based on a simple physical model" Opt. Eng. 40, 2558-2564 (2001).

[5] J. Nicolás, J. Campos and M. J. Yzuel, “Phase and amplitude modulation of elliptic polarization states by nonabsorbing anisotropic elements: application to liquid-crystal devices" J. Opt. Soc. Am. A 19, 1013-1020 (2002).

[6] S. T. Wu and D. K. Yang, Reflective Liquid Crystal Displays (John Wiley at Sons Inc., Chichester, 2005). 
[7] H. Dai, K. Xu, Y. Liu, X. Wang and J. Liu, "Characteristics of LCOS Phase-only spatial light modulator and its applications" Opt. Commun. 238, 269-276 (2004).

[8] T. Ito, T. Shimobaba, H. Godo and M. Horiuchi, “Holographic reconstruction with a $10-\mu \mathrm{m}$ pixel-pitch reflective liquid-crystal display by use of a light-emitting diode reference light" 0pt. Lett. 27, 1406-1408 (2002).

[9] J. Kacperski and M. Kujawinska, "Active, LCoS based laser interferometer for microelements studies" Opt. Express 14, 9664-9678 (2006).

[10] J. E. Wolfe and R. A. Chipman, "Polarimetric characterization of liquid-crystal-on-silicon panels" Appl. Optics 45, 1688-1703 (2006).

[11] A. Márquez, I. Moreno, C. Iemmi, A. Lizana, J. Campos and M. J. Yzuel, "Mueller-Stokes characterization and optimization of a liquid crystal on silicon display showing depolarization" Opt. Express (in press).

[12] A. Hermerschmidt, S. Osten, S. Krüger and Thomas Blümel, "Wavefront generation using a phase-only modulating liquid- crystalbased micro-display with HDTV resolution" Proc. of SPIE 6584, 65840E (2007).

[13] A. Márquez, C. Iemmi, J. Campos and M. J. Yzuel, "Achromatic diffractive lens written onto a liquid crystal display" 0pt. Lett. 31, 392-394 (2006).

[14] A. Márquez, C. Iemmi, J. Campos, J. C. Escalera and M. J. Yzuel, "Programmable apodizer to compensate chromatic aberrations effects using a liquid crystal spatial light modulator" Opt. Express 13, 716-730 (2005).

[15] D. Goldstein, Polarized Light (Marcel Dekker, 2004).

[16] S. Stallinga, "Equivalent retarder approach to reflective liquid crystal displays" J. Appl. Phys. 86, 4756-4766 (1999)

[17] A. Márquez, I. Moreno, J. Campos and M. J. Yzuel, "Analysis of Fabry-Perot interference effects on the modulation properties of liquid crystal displays" 0pt. Commun. 265, 84-94 (2006).

[18] S. Y. Lu and R. A. Chipman, "Interpretation of Mueller matrices based on polar decomposition" J. Opt. Soc. Am. A 13, 1106-1113 (1996). 